\title{
Análisis Termodinámico de las Turbinas de Vapor para los Ciclos Ultracríticos, Supercríticos, Subcríticos y Geotérmicos
}

\author{
Ladislao E. Méndez-Cruz ${ }^{(1)}$, Martín Salazar-Pereyra(1), Angélica E. Bonilla-Blancas ${ }^{(2)}$ y Raúl Lugo-Leyte( ${ }^{(3)}$ \\ (1) Tecnológico de Estudios Superiores de Ecatepec, Posgrado en Eficiencia Energética y Energías Renovables, \\ Av. Tecnológico s/n, Valle de Anáhuac, Ecatepec de Morelos, Estado de México, México. \\ (e-mail: ladislao.eduardo@gmail.com, msalazar@tese.edu.mx) \\ (2) Centro de Tecnología Avanzada, CIATEQ, Parque Industrial ex Hacienda Doña Rosa, Lerma, Estado de México, \\ México. (e-mail: angelica.bonilla@ciateq.mx) \\ (3) Departamento de Ingeniería de Procesos e Hidráulica, Universidad Autónoma Metropolitana - Iztapalapa, Avenida \\ San Rafael Atlixco 186, Col. Vicentina, 09340, Iztapalapa, CDMX, México. (e-mail: Iulr@xanum.uam.mx)
}

Recibido Oct. 9, 2018; Aceptado Dic. 19, 2018; Versión final Mar. 8, 2019, Publicado Ago. 2019

\begin{abstract}
Resumen
En este trabajo se presenta el análisis energético y exergético de las centrales de generación de potencia que utilizan las configuraciones de ciclos de vapor ultracríticos, supercríticos, subcríticos regenerativos y los sistemas geotérmicos con dos y tres cámaras flash. Este análisis se realiza principalmente a las turbinas de vapor para analizar la transformación y la degradación de la energía en cada una de las etapas de expansión en función del grado de recalentamiento y de las irreversibilidades generadas. En las etapas de expansión de presión intermedia que se realizan después del primer y segundo recalentamiento se tienen las eficiencias exergéticas más altas. Sin embargo, para el caso del ciclo supercrítico, la máxima eficiencia se tiene en la segunda etapa de expansión de la sección de alta presión. Las menores eficiencias se tienen en los sistemas geotérmicos debido a su condición de baja entalpía, aunque la disponibilidad de la energía se aprovecha en $42 \%$.
\end{abstract}

\section{Thermodynamic Analysis of Steam Turbines for Ultracritical, Supercritical, Subcritical and Geothermal Cycles}

\begin{abstract}
This paper presents the thermodynamics analysis of the power generation plants that use ultracritical, supercritical, subcritical regenerative steam cycle configurations and geothermal systems with two and three flash steam. This analysis is mainly carried out on the steam turbines to analyze the energy transformation and degradation in each of the expansion stages as function of the degree of overheating and the generated irreversibilities. The greater exergetic efficiencies are obtained in the stages of expansion of intermediate pressure that are done after the first and second reheat. However, for the case of the supercritical cycle the maximum efficiency occurs in the second stage of expansion of the high-pressure section. Geothermal systems have the lowest efficiencies due to their low enthalpy condition, although the availability of energy is used in $42 \%$.
\end{abstract}

Keywords: steam turbines; exergy efficiency; ultracritical; subcritical; geothermal 


\section{INTRODUCCIÓN}

Las turbinas de vapor son las más utilizadas para la generación de energía en plantas: ultracríticas y supercríticas (carboeléctricas), subcríticas, (termoeléctricas convencionales), geotérmicas, ciclos combinados y en sistemas de cogeneración. En México, la capacidad instalada de generación de energía eléctrica con turbinas de vapor representa aproximadamente el $60 \%$. Esta perspectiva depende principalmente de las políticas energéticas que establecen el uso de gas natural y carbón mineral como combustibles. En las carboeléctricas para tener una reducción de inquemados y cenizas se utilizan precipitadores electrostáticos. Así mismo, como parte de la modernización y rehabilitación de las centrales termoeléctricas se les ha realizado el cambio de quemadores para gas natural y ya no utilizar combustóleo. Con estas acciones se garantiza una nueva generación limpia, NGL. De igual forma, una de las alternativas para generar potencia es el recurso geotérmico, que en México se tiene disponible en los estados de Michoacán, Puebla, Baja California Sur entre otros. A nivel mundial, países como China, India, Japón, Rusia, Países Bajos y USA cuentan con una generación de energía eléctrica importante a través de las centrales carboeléctricas, utilizando turbinas de vapor a condiciones supercríticas, Retzlaff y Ruegger, (1996). En la actualidad, estos países siguen incrementando su producción de energía con base al carbón, Yong Li et al (2018). Así mismo, Prieto y Paredes (2019) establecen que las nuevas tecnologías de energía nuclear con reactores de cuarta generación utilizarán ciclos de vapor supercríticos y ultracríticos.

En México, la mayoría de las centrales termoeléctricas operan bajo el funcionamiento de los ciclos Rankine con sobrecalentamiento, recalentamiento y regeneración. Estas plantas trabajan con trenes de calentamiento de seis o siete calentadores de los cuales uno es abierto y funciona como deareador y/o amortiguador. Los ciclos Rankine ultracríticos y supercríticos operan a condiciones superiores al punto crítico del agua. Generalmente se les denomina ciclos Rankine ultracríticos a las configuraciones que incluyen dos recalentamientos y supercríticos a los que sólo incluyen un recalentamiento en la caldera ultracrítica. Este tipo de ciclos se implementan con la finalidad de aumentar la eficiencia energética y la potencia generada con respecto a los ciclos subcríticos. Y finalmente, las centrales geotérmicas que operan en el régimen de vapor saturado húmedo con temperaturas inferiores a los $250^{\circ} \mathrm{C}$; y para incrementar su potencia y eficiencia térmica se pueden colocar 2 o 3 cámaras flash para obtener mayor flujo de vapor seco. Kotas (1985) establece la metodología exergética de evaluación de las turbinas de vapor con multi-etapas de expansión, Dincer et al (2001) también ha realizado estudios exergéticos a plantas de generación de potencia, sin embargo, no muestran las eficiencias exergéticas de las etapas de expansión características de las turbinas de vapor, de acuerdo a la configuración del sistema. Otros autores como Zhigang Zhao et al (2017), Hermann et al (2005), Khaliq et al (2004), Gholam et al (2016), Song et al (2002), han cuantificado las pérdidas de exergía y las irreversibilidades que se producen en una central de generación de potencia específica. En este trabajo se analizan seis configuraciones de plantas de generación de potencia, enfocándose principalmente en las turbinas de vapor en función de la eficiencia isoentrópica, debido a que, a través de ésta se puede considerar de forma global las fallas ocasionadas por factores como corrosión, inestabilidades de flujo, ensuciamientos entre otros (Turgay et al, 2018).

R. Lugo et al. (2009), establecen que en las termoeléctricas convencionales, las turbinas de vapor contribuyen en la destrucción de la disponibilidad de energía en promedio del $4 \%$ y de éstas, el $51 \%$ se tienen en la sección de baja presión con respecto a la energía suministrada por el combustible. Dincer et al (2001) cuantifica $8 \%$ en promedio de pérdidas de disponibilidad de la energía en la turbina de vapor, con base al flujo de calor suministrado al generador de vapor. Salazar-Pereyra (2016) muestra un comparativo termodinámico de primera ley de las centrales ultracríticas y subcríticas, donde se establece la relevancia del criterio de elección de las presiones de recalentamiento para una central ultracrítica. Viera et al (2017) establecen que para minimizar las irreversibilidades asociadas al intercambio de calor en el tren de calentamiento en las centrales supercríticas y ultracríticas se requieren 8 y 10 calentadores, respectivamente, debido a la reducción de las fracciones de vapor extraídas de la turbina y al aumento de la temperatura de precalentamiento. Así mismo, Çetin et al (2018) muestran que la República de Turquía depende en más del $50 \%$ de los combustibles fósiles para la generación de energía eléctrica, por tal motivo presenta un comparativo energético y exergético de dos plantas supercríticas y ultracríticas como posibles propuestas para abastecer la demanda de electricidad a bajo costo y menores impactos al medio ambiente con condiciones de operación límites de 300 bar y $600^{\circ} \mathrm{C}$; obteniendo que la central ultracrítica con doble recalentamiento incremente la eficiencia térmica y exergética en $1.5 \%$ y $1.31 \%$, respectivamente, al compararla con el funcionamiento de una supercrítica. Por otra parte, Salazar-Pereyra et al (2017) y Chamorro et al (2012) muestran la metodología y los valores para encontrar la presión óptima de la primera cámara flash en las configuraciones de las centrales geotérmicas que operan con vapor húmedo.

Así mismo, de acuerdo con Retzlaff y Ruegger, (1996) establecen que, para avanzar en el desarrollo de la tecnología de las turbinas de vapor; la posibilidad de mejorar las eficiencias mecánicas mediante la reducción de las pérdidas aerodinámicas y de fugas a medida que el vapor se expande a través de la 
turbina. El segundo tipo de avance es la mejora en la eficiencia termodinámica al aumentar la temperatura y la presión a la que se opera la turbina. De éste dependerá el tipo turbina, región de operación y el número de calentadores de la línea principal de agua. Por consiguiente, en este trabajo sólo se enfoca al estudio termodinámico con base a la metodología del análisis exergético de las turbinas de vapor de los ciclos ultracríticos, supercríticos y subcríticos Rankine y del sistema geotérmico con una y dos cámaras flash para determinar la eficiencia exergética de expansión y global de la turbina en función de la eficiencia isoentrópica de la turbina.

\section{CICLOS DE VAPOR}

En la Tabla 1 se presentan las condiciones de operación de las centrales de vapor. Estas condiciones de operación se obtienen de los balances térmicos de las centrales que se encuentran actualmente en funcionamiento. En la Fig. 1 se muestra el diagrama de flujo para determinar los parámetros a evaluar de los ciclos de vapor. Con los datos de operación, ambientales y tecnológicos se obtienen los estados termodinámicos y se realizan los balances de masa, energía y exergía y se evalúan los procesos que intervienen. Finalmente se obtiene la eficiencia exergética de las etapas de expansión, globales y de la planta. Las metodologías completas se describen en Salazar-Pereyra et al (2017) y Lugo et al (2009).

Tabla 1: Condiciones de operación de las centrales de vapor.

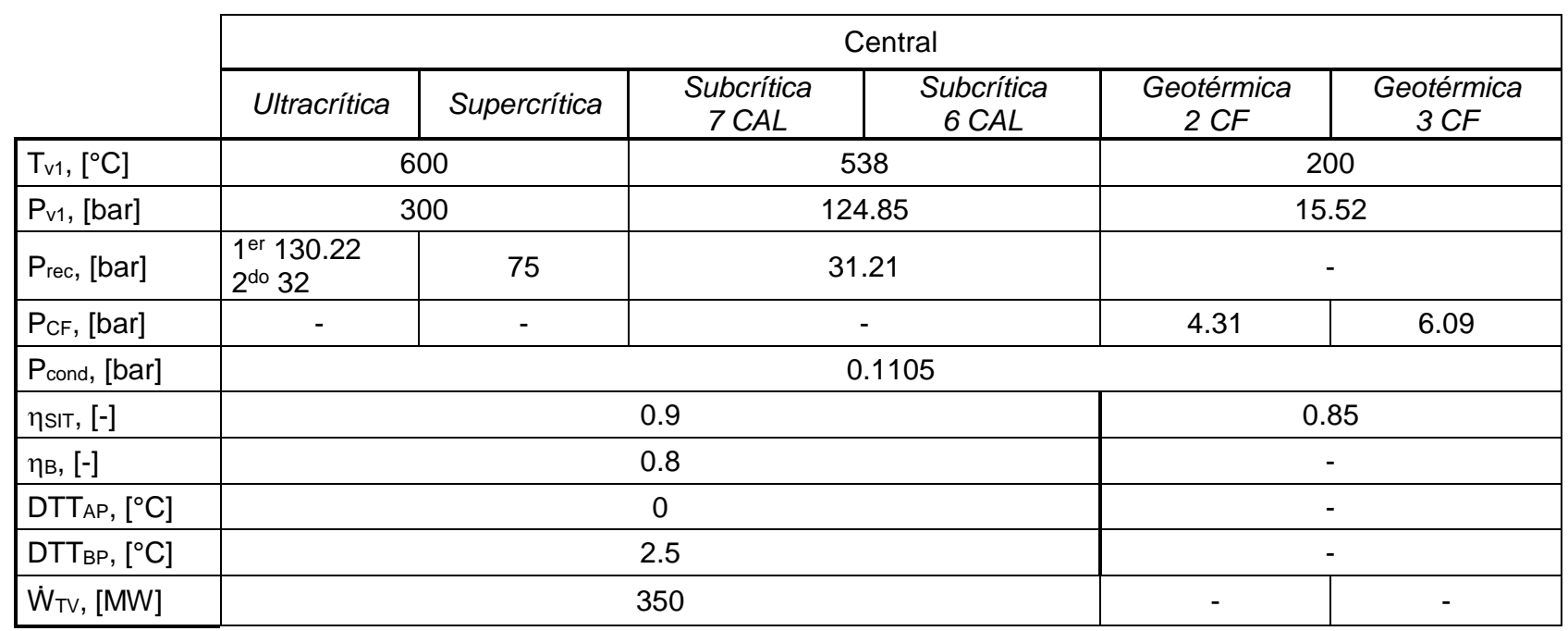

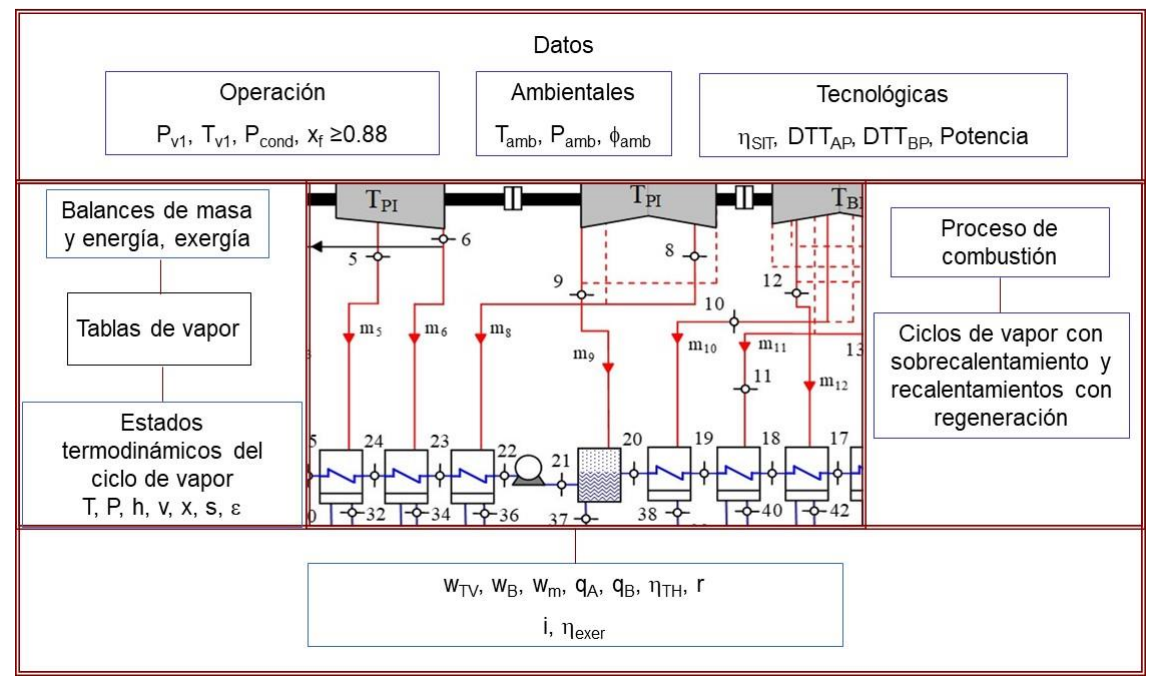

Fig. 1: Diagrama de flujo para evaluar los ciclos de vapor.

En la Fig. 2 se muestran los diagramas temperatura-entropía de los ciclos de vapor ultracríticos, supercríticos, subcríticos y geotérmicos húmedos. El ciclo ultracrítico tiene dos recalentamientos, 11 etapas de expansión y 10 calentadores de línea. El ciclo supercrítico tiene un recalentamiento, 9 etapas de expansión y 8 calentadores de línea. Con respecto a los sistemas geotérmicos, las expansiones de la turbina de vapor se encuentran en la región de vapor húmedo. Asimismo, todos los ciclos tienen una restricción en la operación de la turbina de vapor, en la última etapa de expansión, la calidad del vapor debe 
ser mayor o igual a 0.88 .

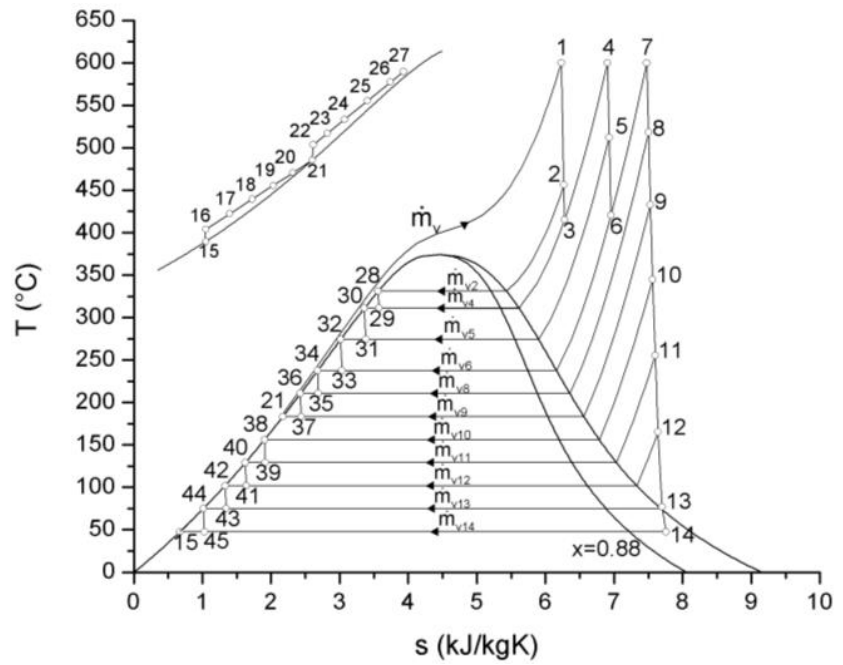

2a) Ultracrítico

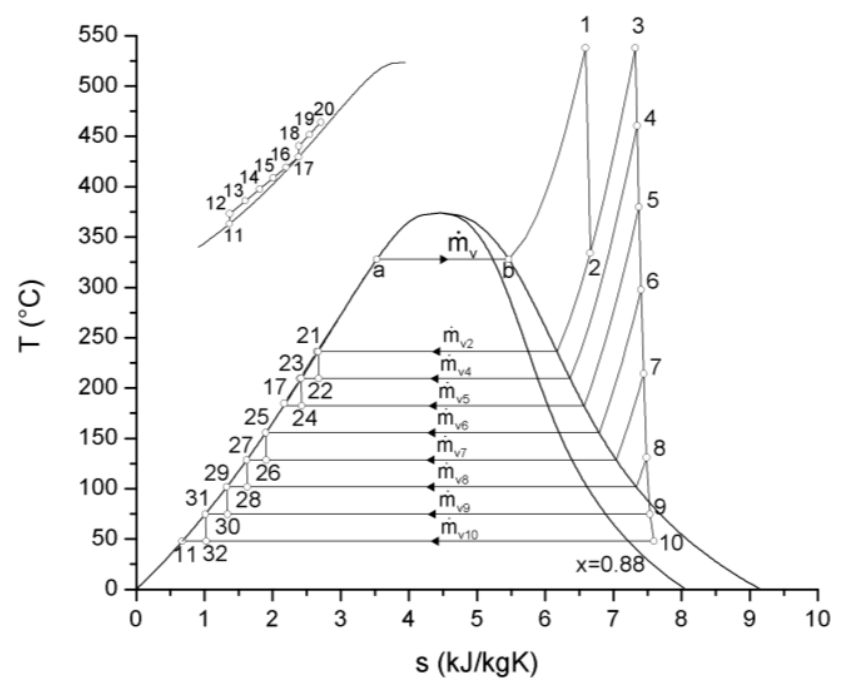

2c) Subcrítico con 7 calentadores de línea

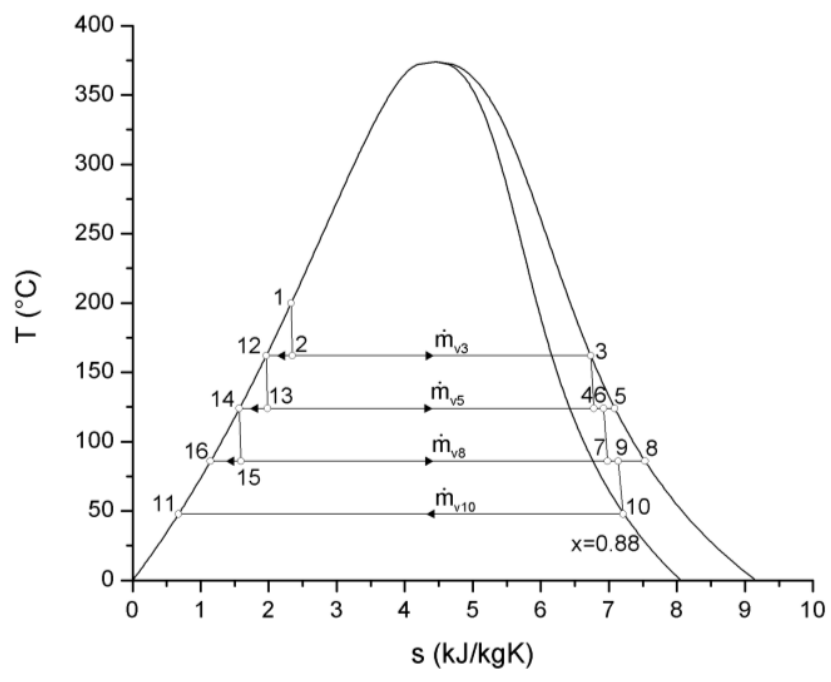

2e) Geotérmico con tres cámaras flash

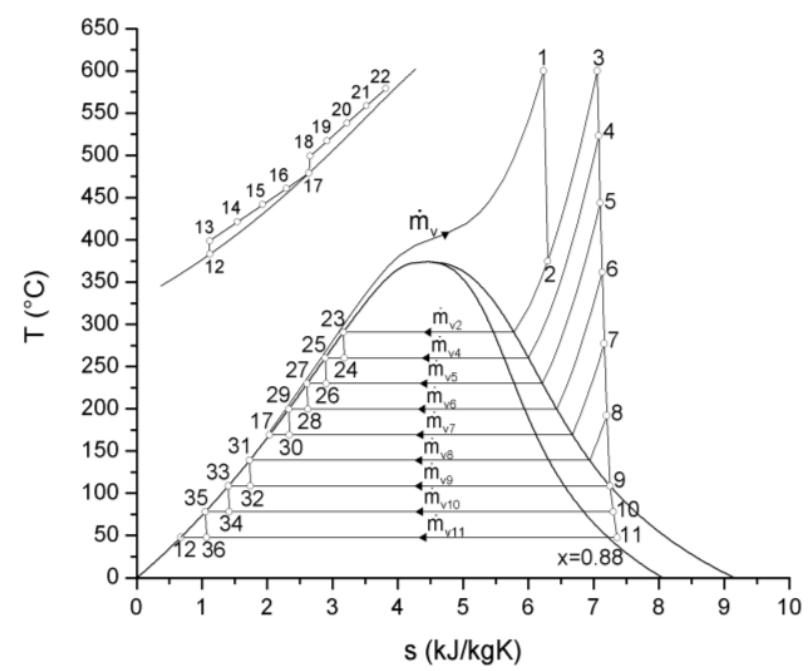

2b) Supercrítico

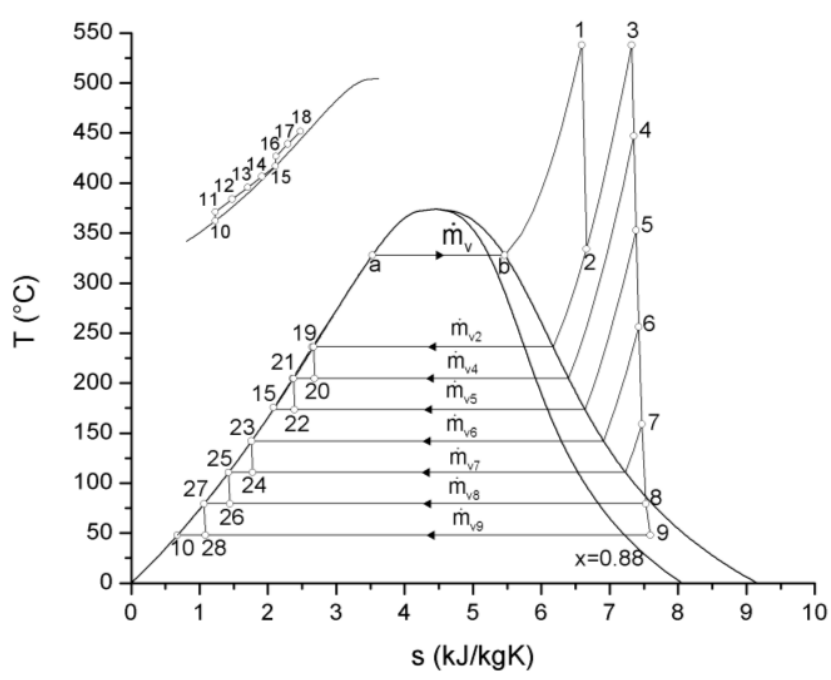

2d) Subcrítico con 6 calentadores de línea

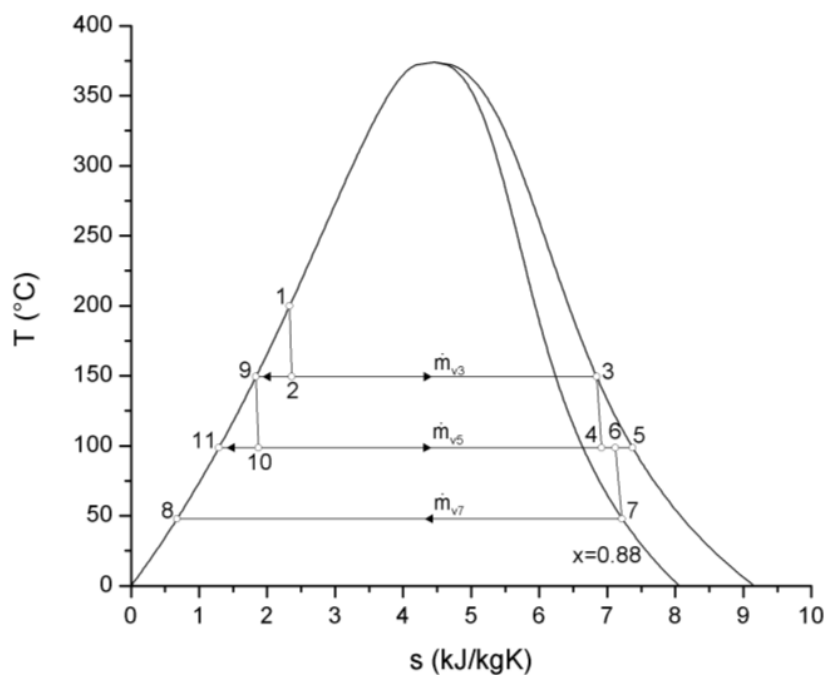

2f) Geotérmico con dos cámaras flash

Fig. 2: Diagramas Temperatura-entropía de los ciclos de vapor y geotérmicos.

Las presiones y temperaturas de las turbinas de vapor de las centrales ultracríticas y supercríticas se encuentran en un intervalo de 241 a 310 bar y de $538^{\circ} \mathrm{C}$ a $600^{\circ} \mathrm{C}$. En las termoeléctricas con la 
configuración de ciclo Rankine con sobrecalentamiento, recalentamiento y precalentamiento de la línea principal de agua, las turbinas a la entrada tienen un rango de operación de presión y temperatura de 120 a 160 bar y de 538 a $560{ }^{\circ} \mathrm{C}$. Así mismo se presentan las configuraciones que los autores previamente analizaron en función del número de calentadores óptimos en la línea de calentamiento del agua principal, con el cual se obtiene la mayor eficiencia térmica. Por otra parte, las condiciones de la temperatura y la fase del fluido geotérmico determinan el tipo de configuración que se debe de instalar, es decir, de vapor seco, cámaras flash y/o ciclos binarios. El intervalo de las temperaturas del vapor está entre 100 y $350^{\circ} \mathrm{C}$.

\section{METODOLOGÍA}

El trabajo de la turbina de vapor por unidad de masa para las configuraciones de los ciclos ultracríticos, supercríticos y subcríticos se expresa de la siguiente manera

$$
w_{T V}=\sum w_{T V A P}+\sum w_{T V P I}+\sum w_{T V B P}
$$

El trabajo específico de la turbina de vapor en las secciones de alta se define de la siguiente forma, con base a la Figura 2 a), b), c) y d)

$$
w_{T V A P 1}=\left(h_{v 1}-h_{v 2}\right)
$$

Para el arreglo ultracrítico, la segunda etapa de expansión de alta presión, está definida entre los estados 2 y 3

$$
w_{T V A P 2}=\left(1-m_{2}\right)\left(h_{v 2}-h_{v 3}\right)
$$

En el ciclo supercrítico, después del recalentamiento se tiene la segunda expansión de alta presión

$$
w_{T V A P 2}=\left(1-m_{2}\right)\left(h_{v 3}-h_{v 4}\right)
$$

El trabajo específico generado en las siguientes secciones de la turbina de vapor, se obtiene con la siguiente expresión de acuerdo al número de etapas adicionales de expansión de intermedia y de baja presión. Por ejemplo, para el ciclo ultracrítico, la ec. (5) se debe de evaluar para 9 etapas de expansión, del estado 4 al 6 y del estado 7 al 14

$$
w_{T v_{i}}=\left(1-m_{2}-\sum_{i=4}^{n} m_{i}\right)\left(h_{v_{n}}-h_{v_{n+1}}\right)
$$

Para los ciclos subcríticos, las etapas de expansión de presión intermedia y de baja se evalúan con la siguiente expresión

$$
w_{T v_{i}}=\left(1-\sum_{i=2}^{n} m_{i}\right)\left(h_{v_{n}}-h_{v_{n+1}}\right)
$$

Donde i-ésimo representa la fracción masa extraída a la turbina; n-ésimo indica el estado termodinámico inicial del proceso de expansión. Esta expresión no aplica para los procesos de recalentamiento de vapor, es decir, $m_{3}=0$ para los ciclos 1 b) c) y d) y para el ultracrítico $1 a$ ), $m_{3}=0$ y $m_{7}=0$. Los ciclos de vapor convencionales y supercríticos presentan en su arreglo dos bombas, alta y baja presión, por consiguiente, el trabajo suministrado a las bombas es

$$
w_{B}=w_{B B P}+w_{B A P}=\left(1-\sum_{i=2}^{n} m_{i}\right)\left(h_{v j}-h_{v j+1}\right)+\left(h_{v k}-h_{v k+1}\right)
$$

Donde i-ésimo implica el número de la fracción de vapor suministrada al deareador; j-ésimo representa el estado a la salida del condensador y k-ésimo es el estado termodinámico de la salida del agua de alimentación principal del deareador o calentador abierto. Por lo tanto, el trabajo motor obtenido del ciclo de vapor ultracrítico es, $w_{m}=w_{T V}-w_{B}$. 
En las centrales geotérmicas se tienen turbinas de vapor de baja presión, en este caso, el trabajo generado está en función de las fracciones de vapor separadas en la cámara flash, y de las presiones de expansión. El trabajo generado en las configuraciones de dos y tres cámaras flash es

$$
\begin{aligned}
& w_{m_{2 C F}}=x_{v 2}\left(h_{v 3}-h_{v 4}\right)+\left[x_{v 2}+\left(1-x_{v 2}\right) x_{v 10}\right]\left(h_{v 6}-h_{v 7}\right) \\
& w_{m_{3 C F}}=x_{v 2}\left(h_{v 3}-h_{v 4}\right)+\left[x_{v 2}+\left(1-x_{v 2}\right) x_{v 13}\right]\left(h_{v 6}-h_{v 7}\right)+\left[\left(1-x_{v 2}\right)\left(1-x_{v 13}\right) x_{v 13}\right]\left(h_{v 9}-h_{v 10}\right)
\end{aligned}
$$

La exergía física específica por estado termodinámico se define de la siguiente forma

$$
\varepsilon_{i}=\left(h_{v_{i}}-h_{0}\right)-T_{0}\left(s_{v_{i}}-s_{0}\right)
$$

Las irreversibilidades internas del proceso de expansión por etapas y considerando un proceso adiabático, y se expresan de la siguiente manera

$$
i_{T v_{i}}=T_{0}\left(s_{v_{i}}-s_{v_{i+1}}\right)
$$

Entonces, el trabajo generado por las turbinas está en función de la exergía de entrada y salida, sin embargo, estos valores son afectados por la irreversibilidad del proceso, en otras palabras, $\Delta \varepsilon=\mathrm{W}_{\mathrm{TV}}+\mathrm{i}$.

La eficiencia isoentrópica de expansión establece una relación entre el incremento de entropía del proceso de expansión y del trabajo perdido que se denomina grado de recalentamiento. Este factor de diseño y de operación de las turbinas de vapor establece la desviación del trabajo máximo disponible que se puede obtener de un flujo de vapor, es decir, al disminuir la eficiencia isoentrópica, se incrementa la entropía y la temperatura al final de la expansión. La eficiencia exergética de expansión relaciona el trabajo generado por la turbina en función de la exergía suministrada. Kotas (1985) desarrolla la metodología exergética para obtener la eficiencia exergética en cada etapa de expansión en función de ๆsıт, r, i

$$
\eta_{\text {exerg, } T V}=\frac{\eta_{S I T}}{\frac{i_{T V}}{r}+\eta_{S I T}\left(1-\frac{i_{T V}}{r}\right)}
$$

donde, $r$ es el grado de recalentamiento y es la diferencia de entalpías del estado real y el estado isoentrópico al final de cada etapa de expansión de la turbina, es decir, $r=\left(h_{v i}-h_{v i s}\right.$ ). Por lo tanto, la eficiencia exergética total de expansión de la turbina de vapor es

$$
\eta_{\text {exerg }_{T V}}=\frac{1}{\Delta \varepsilon_{\text {total }}} \sum_{i=1}^{n} \eta_{\text {exerg }_{T V, i}}\left(\Delta \varepsilon_{T V, i}\right)
$$

donde $\Delta \varepsilon_{\text {total }}=\sum \Delta \varepsilon_{T V A P}+\sum \Delta \varepsilon_{T V P I}+\sum \Delta \varepsilon_{T V B P}$

Finalmente, la eficiencia térmica y exergética de los ciclos de vapor se determinan a partir de las siguientes expresiones

$$
\begin{aligned}
& \eta_{\text {th }}=\frac{\dot{W}_{m}}{\dot{m}_{v} q_{\text {sum }}} \\
& \eta_{\text {exerg }}=\frac{\dot{W}_{m}}{\dot{m}_{c} P C l\left(1-\frac{T_{0}}{T_{\text {fa }}}\right)}
\end{aligned}
$$

El $P C l$ se obtiene a partir de la composición del gas natural, $T_{0}$ es la temperatura ambiente y $T_{f a}$ es la temperatura de flama adiabática correspondiente al combustible empleado. Para el caso de las centrales 
geotérmicas, la eficiencia térmica y exergética se compara con base a las condiciones del recurso geotérmico, es decir del vapor húmedo. (Salazar-Pereyra et al, 2017).

$$
\begin{aligned}
& \eta_{t h}=\frac{w_{m, C F}}{h_{v 1}-h_{0}} \\
& \eta_{\text {exerg }}=\frac{w_{m, C F}}{\left(h_{v_{1}}-h_{0}\right)-T_{0}\left(s_{v 1}-s_{0}\right)}
\end{aligned}
$$

\section{RESULTADOS Y DISCUSIÓN}

En la Fig. 3 se muestran los diagramas exergía-entalpía para las configuraciones de los ciclos ultracrítico, supercrítico, subcríticos con 7 y 6 calentadores y los geotérmicos con dos y tres cámaras flash $(3 \mathrm{a}, 3 \mathrm{~b}, 3 \mathrm{c}$, $3 \mathrm{~d}, 3 \mathrm{e}$ y $3 \mathrm{f}$ ), respectivamente. Al comparar el comportamiento de los ciclos se muestra que, al incrementar la presión del vapor a la entrada de la turbina de 124 a 300 bar, no aumenta en mayor porcentaje la disponibilidad de la energía en el estado 1, es decir, sólo se obtienen $100 \mathrm{~kJ} / \mathrm{kg}$ de diferencia. Sin embargo, la disponibilidad de la energía si se incrementa en los estados de las siguientes etapas de expansión. Para el ciclo ultracrítico se tienen los estados termodinámicos de 1 a 9 , con la calidad de la energía mayor a 1100 $\mathrm{kJ} / \mathrm{kg}$ que se utilizan en 6 etapas de expansión; por otra parte, el ciclo supercrítico tiene los primeros 5 estados por encima de este valor y se utilizan en 3 etapas de expansión. En el caso de los ciclos subcríticos se muestran sólo 4 estados y dos etapas de expansión, donde se puede obtener mayor generación de trabajo útil. En estas etapas de los ciclos se aprovecha entre el $40 \%$ al $50 \%$ de la energía para generar trabajo útil. En las etapas de expansión de baja presión (las 3 últimas etapas), se tienen valores de exergía menores a $750 \mathrm{~kJ} / \mathrm{kg}$, y es donde se muestran las mayores pérdidas de trabajo útil, al comparar los valores de exergía y de entalpía, es decir en promedio se puede aprovechar el $25 \%$ de la energía para generar trabajo. Con respecto a la línea de bombeo no se muestran grandes cambios en la exergía debido a su condición de estado líquido y de incompresibilidad.

En los arreglos geotérmicos con dos y tres cámaras flash, se muestra que la cantidad de energía y la exergía se incrementan en $380 \%$ en el estado 3 , (763 kJ/kg y $812 \mathrm{~kJ} / \mathrm{kg}$, respectivamente), con base al valor del recurso geotérmico, estado 1 ; es en la separación de fases de la cámara flash donde se incrementa el valor de la energía latente del vapor, así como su exergía; de esta manera se produce mayor trabajo de expansión en las etapas de la turbina; también se muestra que existe un aumento de la exergía en promedio de $15 \mathrm{~kJ} / \mathrm{kg}$ a la entrada de la segunda y tercer expansión de las turbinas de vapor, a causa de la mezcla del vapor de la etapa anterior y la fracción de vapor obtenida de la siguiente cámara flash.

En las Figuras 4 a 8 se muestran las eficiencias exergéticas de las etapas de expansión de las turbinas de los ciclos de vapor y geotérmicos. Las turbinas de vapor industriales generalmente tienen tres secciones: alta, media y baja presión; y de acuerdo con las extracciones de vapor se establecen las etapas, definiendo la presión y temperatura del vapor a la entrada y la salida del vapor sobrecalentado y/o húmedo (Ponce y Valderrama, 2016). En la Figura 4 se muestra que la turbina de vapor que opera a condiciones ultracríticas tiene dos etapas de expansión de alta, estados 1 a 3; sin embargo, las etapas que tienen mayor eficiencia exergética son las de presión intermedia PI3 y Pl1, estados 7 a 8 y de 4 a 5 , respectivamente. Estas expansiones se tienen después de los dos recalentamientos del vapor, aunque la mayor eficiencia exergética se tiene después del segundo recalentamiento. De la Fig. 1a) se muestra que el sobrecalentamiento y los recalentamientos del vapor son a la misma temperatura, pero diferente presión. Comparando las Figuras 4, 6 y 7, se encuentra que las etapas de mayor aprovechamiento de la exergía, es la sección de presión intermedia, etapa PI1, que corresponde a los estados después del recalentamiento estados 3 a 4. Esta etapa es la que presenta menores irreversibilidades, debido al comportamiento del vapor sobrecalentado a estas presiones y de la relación de presiones de la expansión que genera menos entropía. Los valores de las eficiencias exergéticas de las etapas de expansión de alta presión generalmente se ubican después de las de presión intermedia, debido a la relación de presiones entre la presión del vapor de entrada y la del primer recalentamiento, donde $P_{2}=0.25 P_{1}$. Para la turbina a condiciones supercríticas, la expansión equivalente es la AP2, que también corresponde a la de máxima eficiencia exergética.

La disminución de la eficiencia isoentrópica se ocasiona en las turbinas por el desgaste, erosión y las horas de operación acumuladas, lo que representa una menor capacidad de aprovechamiento de la cantidad y calidad de la energía. En general, en la sección de presión intermedia de las turbinas de vapor, las eficiencias exergéticas de expansión disminuyen aproximadamente $0.5 \%$, por cada $1 \%$ que disminuye la 


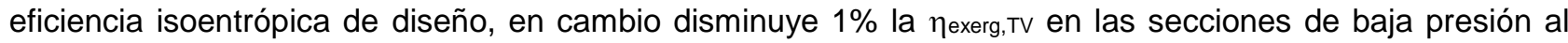
disminuir la $\eta$ sit en $1 \%$, Figuras 4, 5 y 6 . Es decir, que el grado de recalentamiento en las etapas de baja presión contribuye en mayor proporción al incremento de entropía y por consiguiente a las irreversibilidades.

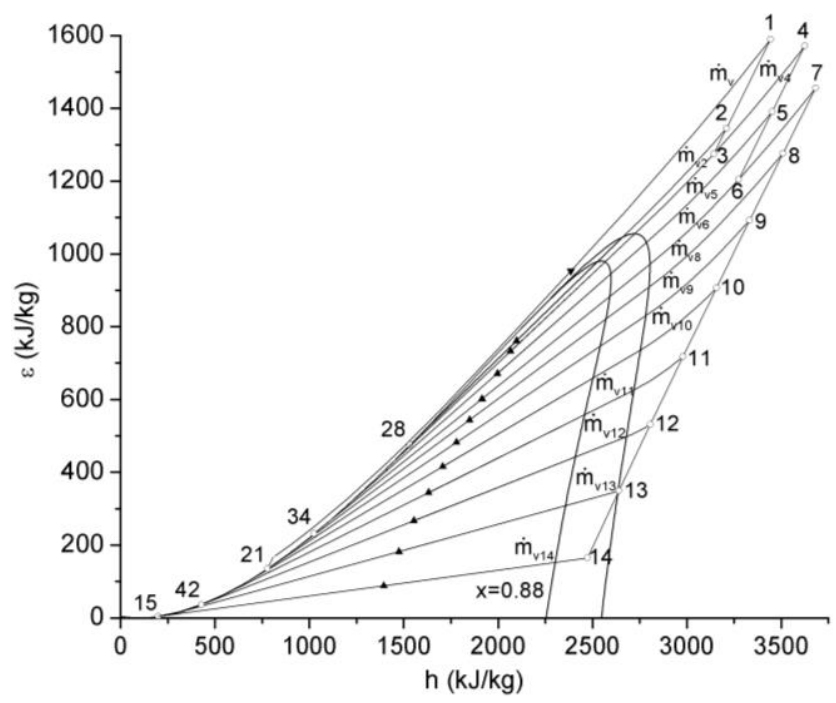

3a) Ultracrítico

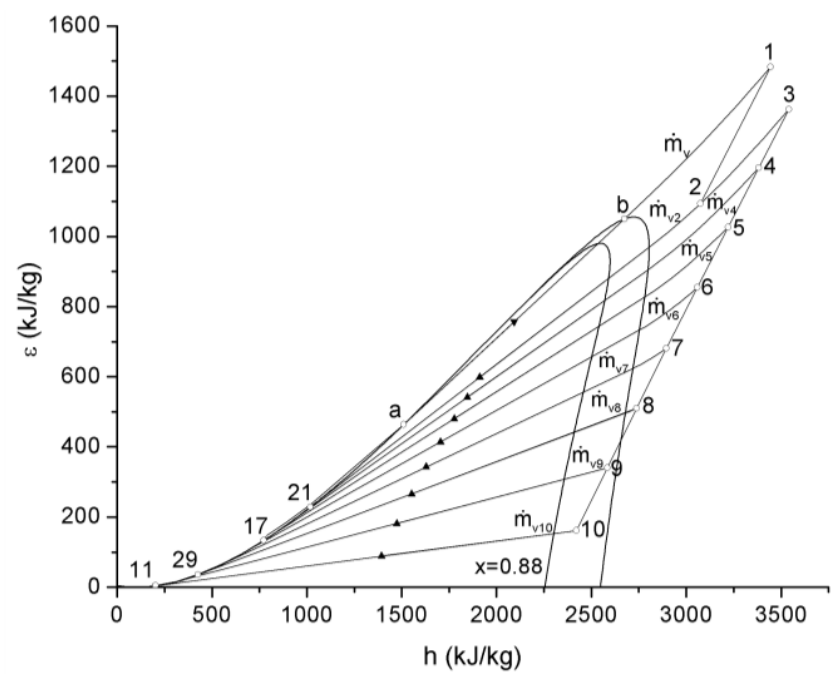

3c) Subcrítico con 7 calentadores de línea

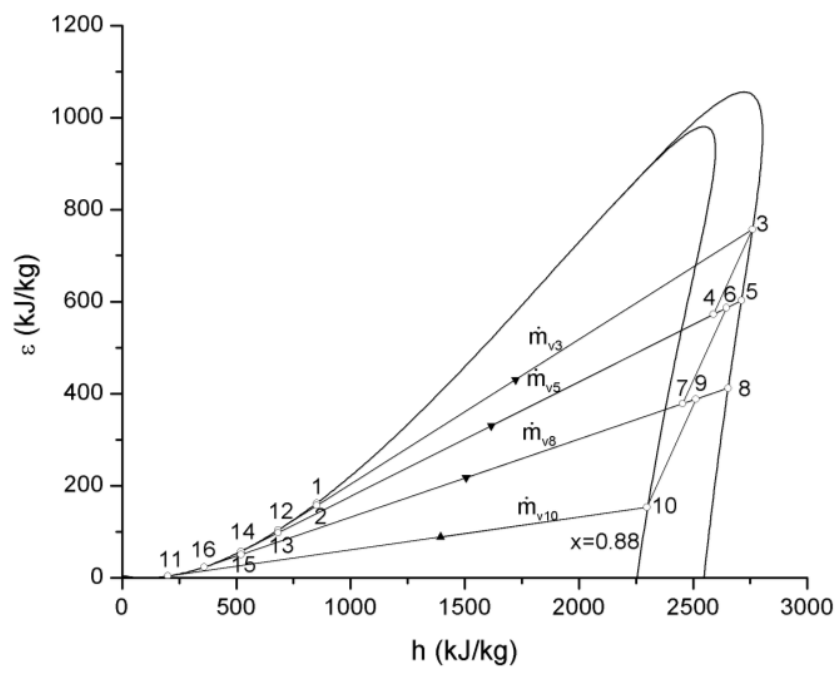

3e) Geotérmico con tres cámaras flash

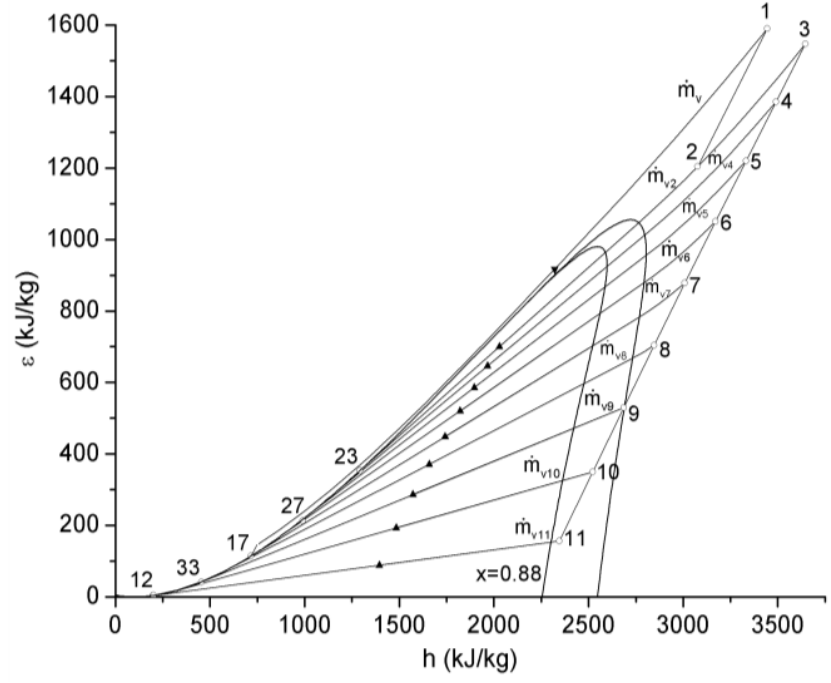

3b) Supercrítico

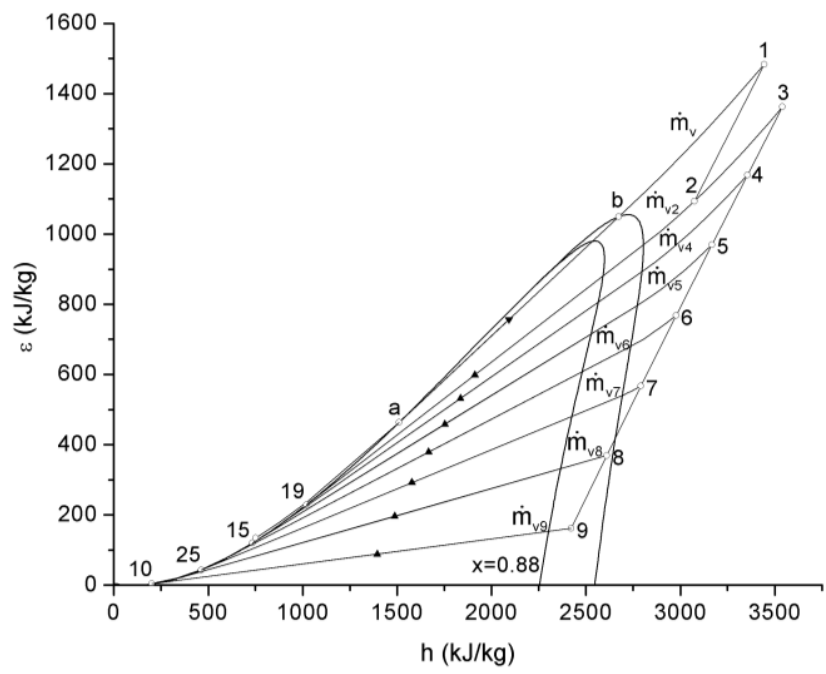

3d) Subcrítico con 6 calentadores de línea

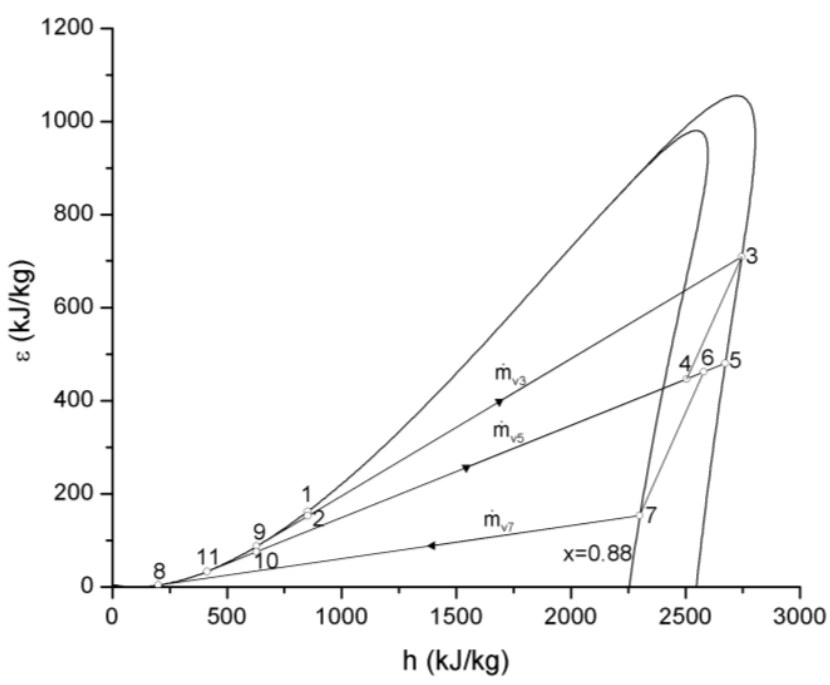

3f) Geotérmico con dos cámaras flash

Fig. 3: Diagramas Exergía-entalpía de los ciclos de vapor y geotérmicos. 
Para el caso de las centrales subcríticas que operan a las mismas condiciones de operación y atmosféricas con diferente número de calentadores de línea de agua principal, la eficiencia exergética de la primera y última etapa de expansión son coincidentes; sin embargo, las etapas de baja presión difieren en mayor proporción al disminuir el número de extracciones y de expansiones, esto se debe al incremento de la relación de expansión en estas etapas y así su entropía, Fig. 7. De acuerdo con R. Lugo et al (2009), el número de calentadores máximo que se pueden instalar en una central térmica subcrítica regenerativa es de 7 , debido a que, con un número mayor de calentadores, la eficiencia térmica del ciclo tiene un incremento menor al $1 \%$, siendo este el objetivo de la regeneración. Algunas de las centrales de ciclos combinados e híbridas presentan arreglos en su ciclo de vapor con menos de 6 calentadores para evitar colocar más intercambiadores de calor en la caldera de recuperación de calor (Srinivas et al, 2014).

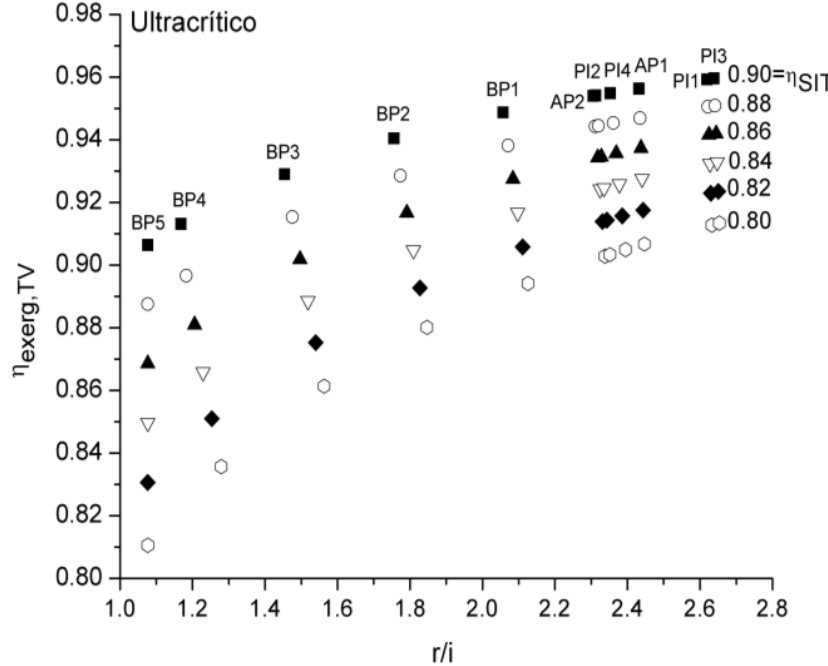

Fig. 4: Variación de la ๆsıt para el arreglo ultracrítico.

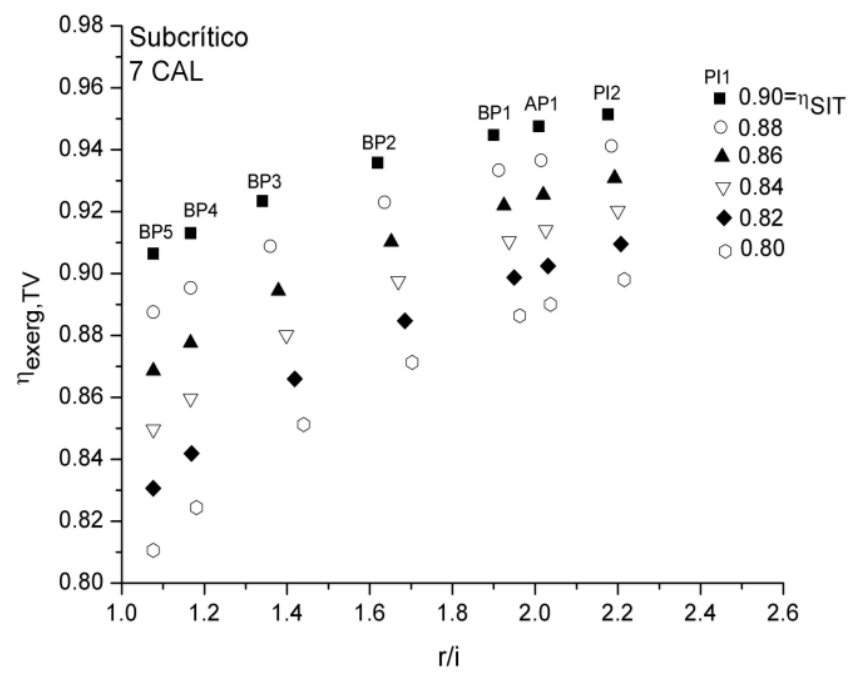

Fig. 6: Variación de la nsıт para el arreglo subcrítico 7 calentadores.

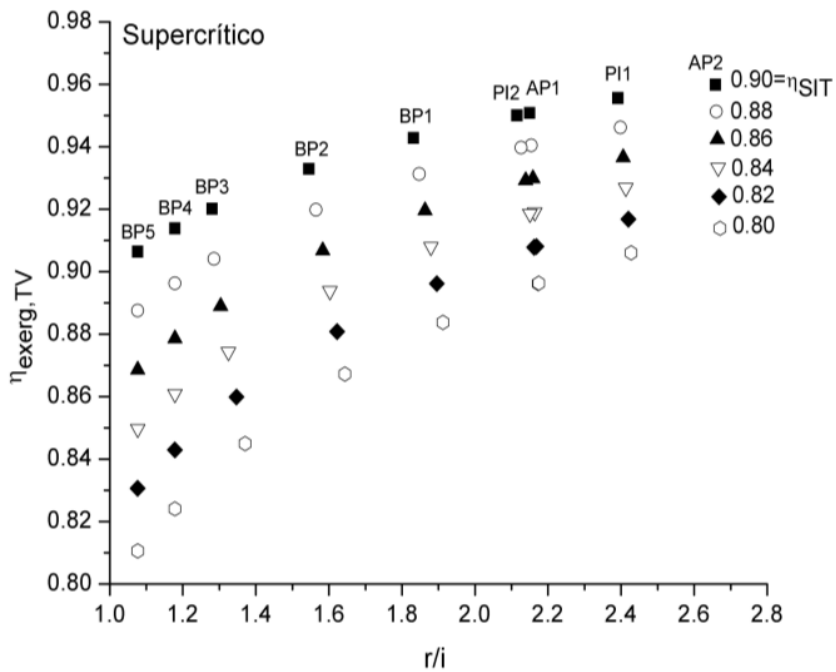

Fig. 5: Variación de la ๆsıt para el arreglo supercrítico.

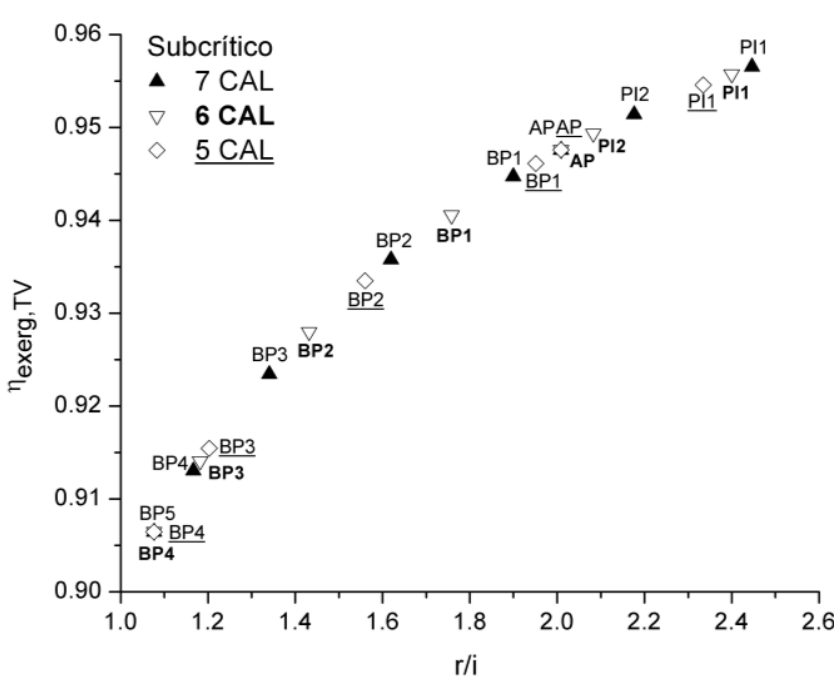

Fig. 7: 包erg,tv en función de r/i para los arreglos subcríticos de 7 hasta 5 calentadores en línea.

Las turbinas de vapor utilizadas en las centrales geotérmicas tienen condiciones de operación de baja entalpía y por consiguiente sólo una sección de baja presión. En la Fig. 8 se muestra que las máximas relaciones $\mathrm{r} / \mathrm{i}$ que se presentan son de 1.375 y con eficiencias exergéticas menores comparadas con las obtenidas con los ciclos subcríticos. Esto se debe principalmente a las condiciones de operación con vapor húmedo que disminuyen las eficiencias isoentrópicas de diseño y de operación. Sin embargo, las eficiencias exergéticas globales de la turbina de vapor del sistema geotérmico son menores en un $2.4 \%$ en promedio con respecto a los ciclos de vapor, Fig. 9. La eficiencia exergética para el caso del ciclo de vapor ultracrítico es la más alta con $94.3 \%$, y para la configuración supercrítica y los dos subcríticos tienen en promedio una eficiencia de $93.6 \%$.

La eficiencia térmica y exergética del ciclo de vapor ultracrítico es mayor al supercrítico en $1 \%$ y $2 \%$, respectivamente y con relación a los ciclos subcríticos es mayor en $5 \%$ y $6 \%$, respectivamente. Los ciclos 
ultracríticos presentan las mayores eficiencias, debido al incremento de la presión y temperatura de entrada del vapor, así mismo la implementación de un segundo recalentamiento. Por otra parte, los ciclos geotérmicos con 3 y 2 cámaras flash muestran las menores eficiencias térmicas y exergéticas, existe claramente una diferencia entre estos parámetros, debido a que las eficiencias exergéticas son superiores a $40 \%$, mientras que las eficiencias térmicas son menores al 10\%, Fig. 10. Sin embargo, el recurso geotérmico no tiene un costo de insumo, por lo tanto, la potencia obtenida es ganancia directa de la planta, sin considerar el proceso de secado del vapor. Así mismo, también el factor de operar en la región de vapor húmedo disminuye la capacidad de generación de trabajo.

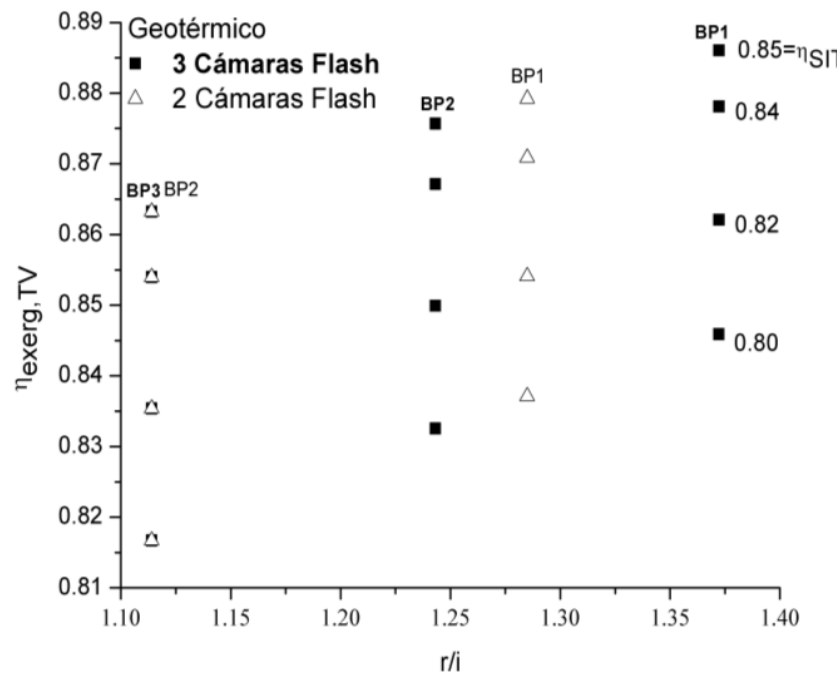

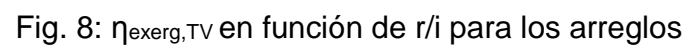
geotérmicos de 3 y 2 cámaras flash

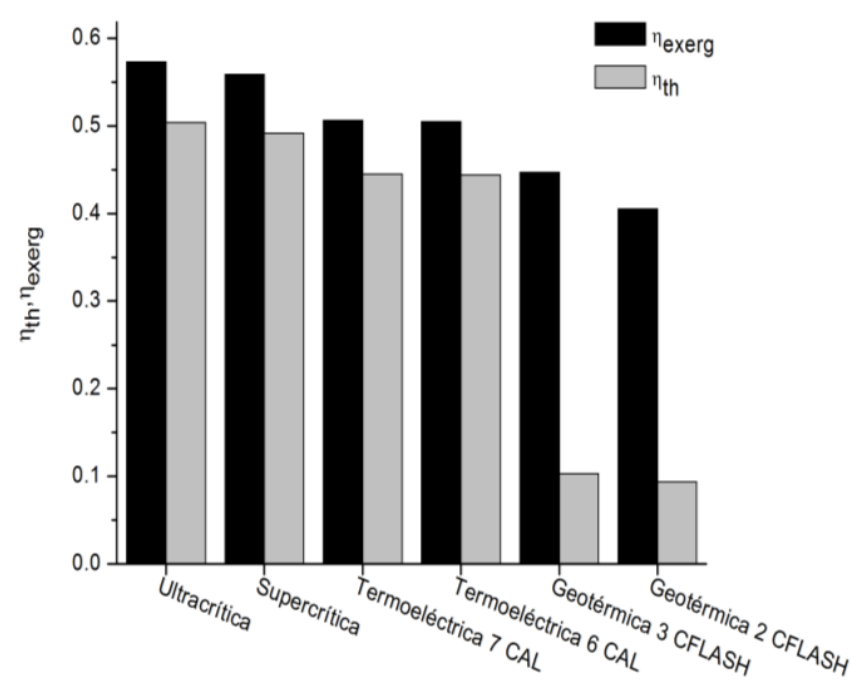

Fig. 10: Eficiencia exergética y eficiencia térmica para los diferentes ciclos de vapor.

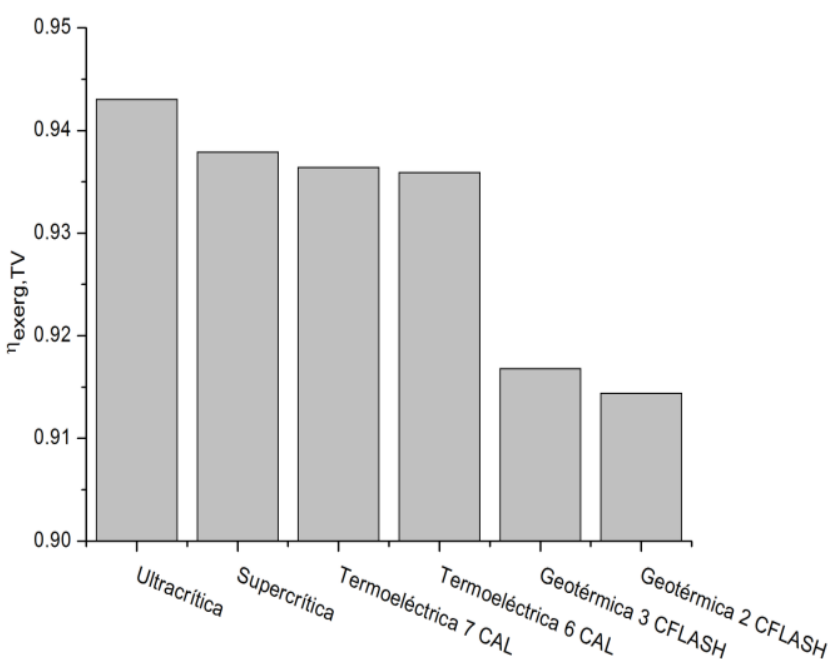

Fig. 9: Eficiencia exergética total de expansión para los diferentes ciclos de vapor.

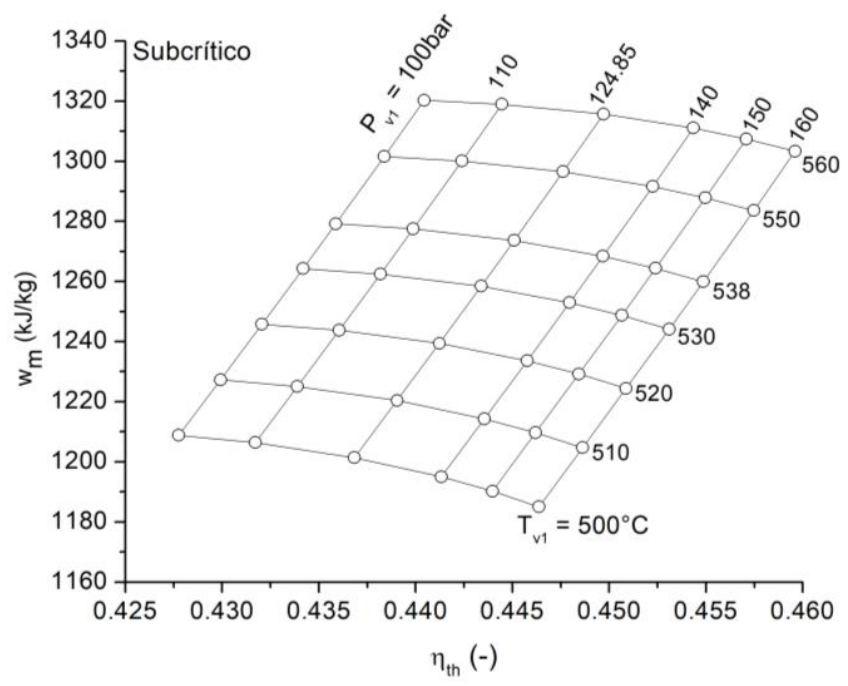

Fig. 11: Trabajo motor y eficiencia térmica en función de las condiciones de entrada de la turbina subcrítica.

En las Figuras 11 a 13 se muestra el trabajo útil y la eficiencia térmica de los ciclos de vapor en función de las condiciones de entrada de la turbina de vapor. El mayor trabajo motor y eficiencia térmica se obtiene en el ciclo ultracrítico en comparación a las otras dos configuraciones. La eficiencia térmica se incrementa $5 \%$ en relación con la mejor configuración de ciclo subcrítico y en 1\% con relación al supercrítico. Sin embargo, el ciclo supercrítico después de una $P_{v 1}=270$ bar y $T_{v 1}=570^{\circ} \mathrm{C}$, en la última etapa de expansión de la turbina se tiene una calidad menor a 0.88 , lo que implicaría operar de forma inestable. Con respecto al trabajo generado en la turbina de vapor ultracrítica se incrementa en un $5 \%$. En los diagramas Sankey de las Figuras 14 a 16 se presenta otra forma de evaluar la eficiencia exergética del ciclo de vapor, ésta se cuantifica por medio de las irreversibilidades en los equipos que componen al sistema de generación de potencia. Por efecto de incrementar la presión en la caldera ultracrítica, se disminuye las irreversibilidades en la transferencia de calor, 6.2 puntos porcentuales con respecto al ciclo subcrítico y 1.2 en relación con el supercrítico. Así mismo, disminuyen las irreversibilidades en el tren de calentamiento de la línea de agua, 
esto se debe a la mayor disponibilidad de la energía del vapor extraído de las turbinas. Sin embargo, se incrementan las irreversibilidades en la turbina supercrítica, esto se debe a la mayor generación de trabajo, como se muestra en las Figuras 11 a 13.

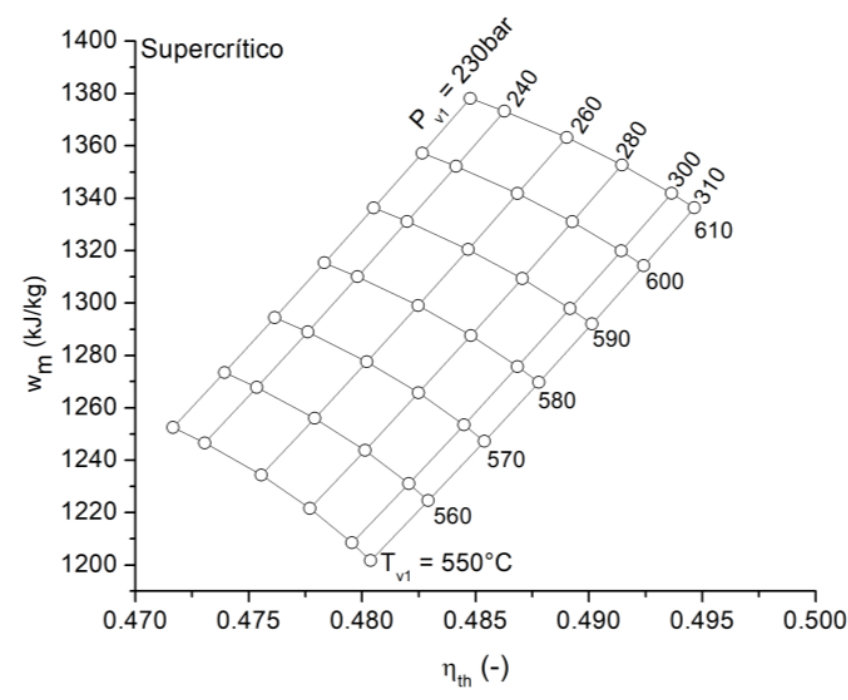

Fig. 12: Trabajo motor y eficiencia térmica en función de las condiciones de entrada de la turbina supercrítica.

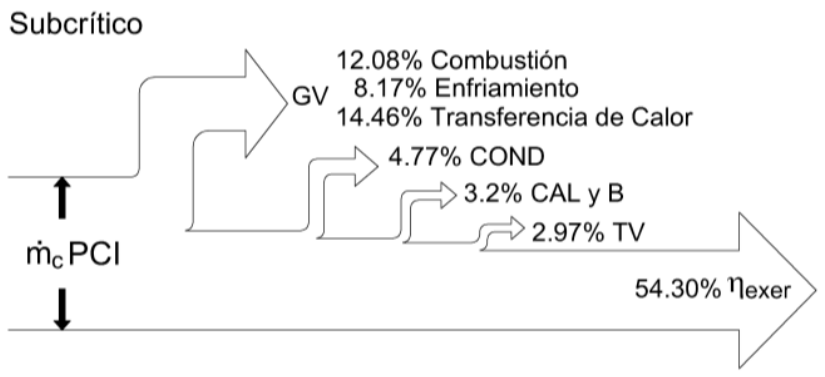

Fig. 14: Diagrama de Sankey para el ciclo subcrítico con 7 calentadores.

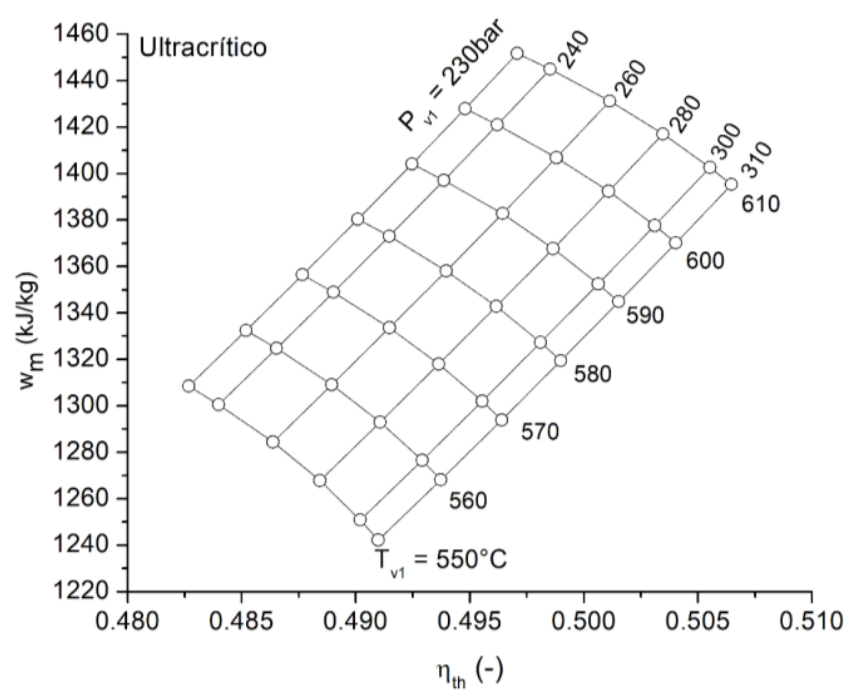

Fig.13: Trabajo motor y eficiencia térmica en función de las condiciones de entrada de la turbina ultracrítica.

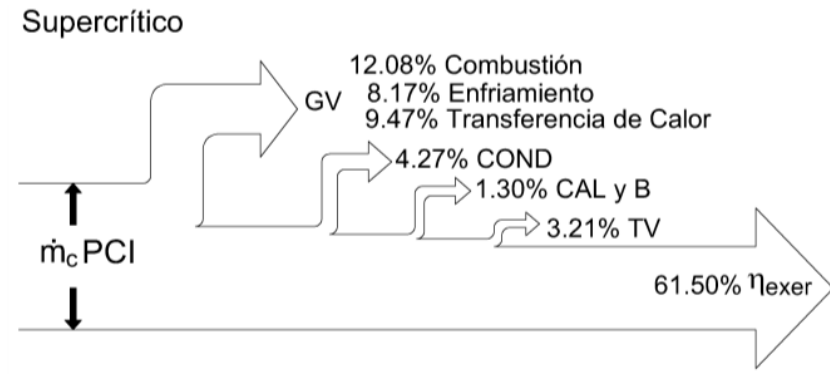

Fig. 15: Diagrama de Sankey para el ciclo supercrítico con 8 calentadores.

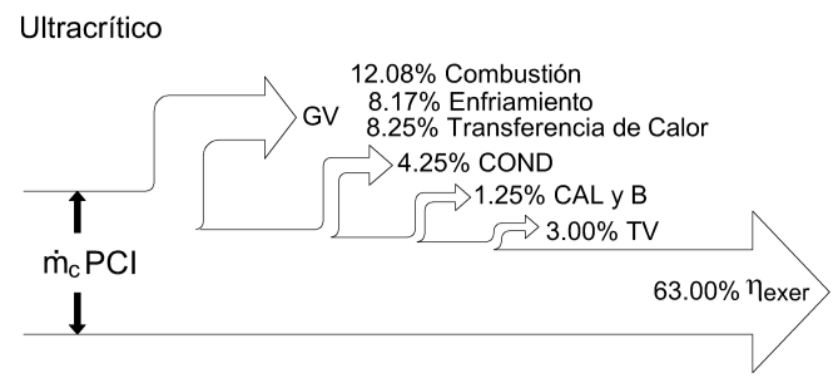

Fig.16: Diagrama de Sankey para el ciclo ultracrítico con 10 calentadores.

\section{CONCLUSIONES}

Los sistemas de generación de energía con ciclos de vapor supercríticos y ultracríticos son los que presentan el mejor comportamiento de acuerdo con el análisis energético y exergético. La configuración de estos ciclos es más robusta lo que permite tener un mayor número de etapas en la turbina de vapor, reduciendo la relación de presiones de las etapas, lo que establece una menor generación de entropía en las expansiones; incrementando el trabajo generado y disminuyendo la degradación de la energía. Además, para México resulta una opción viable debido a que se puede quemar carbón mineral disminuyendo los costos de generación en comparación de los ciclos subcríticos con regeneración que son menos eficientes a los supercríticos y que operan con gas natural. Con respecto a la generación de energía con base al recurso geotérmico se tiene una eficiencia térmica inferior al 10\%, sin embargo, la disponibilidad para generar 
trabajo útil se aprovecha al máximo debido al uso de las cámaras flash. Por otra parte, al disminuir la eficiencia isoentrópica de la turbina de vapor en 1\%, en promedio para todos los arreglos se tiene que la eficiencia exergética disminuye en $0.75 \%$. Así mismo, la máxima relación de r/i para las turbinas de vapor es de 2.7, y es donde se obtiene la máxima eficiencia exergética de las etapas de expansión. Con respecto a la operación de las plantas supercríticas es recomendable implementar un segundo recalentamiento para incrementar la calidad del vapor y así mejorar la seguridad en la operación de la turbina en la última etapa de expansión de baja presión.

\section{AGRADECIMIENTOS}

Al Consejo Nacional de Ciencia y Tecnología, CONACyT, por la beca económica para la obtención del grado de maestro en Eficiencia Energética y Energías Renovables del Ing. Ladislao Eduardo Méndez Cruz.

\section{REFERENCIAS}

Cetin, B., y E. Ozen, Comparative energy and exergy analysis of a power plant with super-critical and sub-critical, http://dx.doi.org/10.18186/thermal.465644, Journal of Thermal Engineering, 4 (6), 2423-2431 (2018)

Chamorro, C.R., M.E. Mondéjar y otros 4 autores, World geothermal power production status: Energy, environmental and economic study of high enthalpy technologies, DOI: 10.1016/j.energy.2011.06.005, Energy, 42(1), 10-18 (2012)

Dincer, I. y H. Al-Muslim, Thermodynamic analysis of reheat cycle steam power plants, https://doi.org/10.1002/er.717, Int. J. Energy Res, 25(8), 727-739 (2001)

Gholam R.A. y T. Davood, Energy and exergy analysis of Montazeri Steam Power Plant in Iran, https://doi.org/10.1016/j.rser.2015.11.074, Renewable and Sustainable Energy Reviews, 56, 454-463 (2016)

Hermann, W.A., Quantifying global exergy resources, https://doi.org/10.1016/j.energy.2005.09.006, Energy, 31(12), 1685-1702 (2006)

Khaliq, A. y S.C. Kaushik, Second-law based thermodynamic analysis of Brayton/Rankine combined power cycle with reheat, https://doi.org/10.1016/j.apenergy.2003.08.002, Applied Energy, 78(2), 179-197 (2004)

Kotas, T.J., The exergy method of thermal plant analysis, $1^{\mathrm{a}}$ Ed., 99-104, Ed. Butterworth, Londres (1985)

Lugo, R., M. Salazar y otros 3 autores, Análisis exergético de una central termoeléctrica, Científica, 13(1), 17-25 (2009)

Moh'd A, B.M. Tashtoush y A. Jaradat, Modeling and simulation of thermoelectric device working as a heat pump and an electric generator under Mediterranean climate, DOI: 10.1016/j.energy.2015.06.090, Energy, 90, 1239-1250 (2015)

Ponce, V.C. y J.O. Valderrama, Análisis de la Influencia de la Radiación Solar en una Planta de Ciclo Combinado con Colectores Solares Integrados mediante Simulación, https://dx.doi.org/10.4067/S0718-07642016000300014, Información Tecnológica, 27(3), 151-160 (2016)

Prieto, G.A. y E.G. Paredes, Linear and Non-linear Stability Analysis in Boiling Water Reactors, 1-53, Ed. Elsevier, United Kingdom (2019)

Retzlaff, K.M. y A.W. Ruegger, Steam Turbines for Ultrasupercritical Power Plants, GE Power Systems, Schenectady, NY, GER-3945a, 1-18 (1996)

Salazar-Pereyra M., A. Mora-Ortega y otros 3 autores, Parametric analysis of the geothermal power: Dry-Steam, flash steam and hybrid cycle, DOI: https://doi.org/10.15446/dyna.v84n203.66126, Dyna, 84(203), 273-282 (2017)

Salazar-Pereyra, M., R. Lugo-Leyte y otros 2 autores, Thermodynamic Analysis of Supercritical and Subcritical Rankine Cycles, doi:10.1115/GT2016-57814, In ASME Turbo Expo 2016: Turbomachinery Technical Conference and Exposition, pp. V008T26A041-V008T26A041, ASME (2016)

Song, T. W., L.J. Sohn y otros 2 autores, Exergy-based performance analysis of the heavy-duty gas turbine in part-load operating conditions, https://doi.org/10.1016/S1164-0235(01)00050-4, Exergy, 2(2), 105-112 (2002)

Srinivas, T. y V.B. Reddy, Comparative studies of augmentation in combined cycle power plants, https://doi.org/10.1002/er.3139, J. Energy Res., 38(9), 1201-1213 (2014)

Turgay, K., S.O. Salim, Conventional and advanced exergy analyses of a marine steam power plant, https://doi.org/10.1016/j.energy.2018.08.119, Energy, 163, 392-403 (2018)

Vieira, A. F., N. Fraidenraich, y L. De Souza, Optimum Efficiency Analysis of Regenerative Cycle with Feed Water Heaters, doi: 10.4236/jpee.2017.58004, Journal of Power and Energy Engineering, 5, 45-55 (2017)

Yong Li, Y.W. y otros 3 autores, Modeling for the performance evaluation of $600 \mathrm{MW}$ supercritical unit operating No.0 high pressure heater, https://doi.org/10.1016/j.energy.2018.01.103, Energy, 149, 639-661 (2018)

Zhigang Zhao, S.S., y otros cinco autores, Exergy analysis of the turbine system in a $1000 \mathrm{MW}$ double reheat ultrasupercritical power plant, https://doi.org/10.1016/j.energy.2016.12.072, Energy, 119, 540-548 (2017) 